\title{
Stability and robust stability of linear time-invariant delay differential-algebraic equations
}

\author{
Nguyen Huu Du* Vu Hoang Linh* Volker Mehrmann ${ }^{\dagger \ddagger}$ \\ Do Duc Thuan $\S$
}

June 23, 2013

\begin{abstract}
Necessary and sufficient conditions for exponential stability of linear time invariant delay differential-algebraic equations (DDAEs) are presented. The robustness of this property is studied when the equation is subjected to structured perturbations and a computable formula for the structured stability radius is derived. The results are illustrated by several examples.
\end{abstract}

Keywords: delay differential-algebraic equation, strangeness-free DAE, exponential stability, spectral condition, restricted perturbation, stability radius.

Mathematics Subject Classifications: 06B99, 34D99, 47A10, 47A99, 65 P99.

\footnotetext{
*Faculty of Mathematics, Mechanics and Informatics, Vietnam National University, 334, Nguyen Trai Str., Thanh Xuan, Ha Noi, Vietnam, email: \{dunh,linhvh\}@vnu.edu.vn

${ }^{\dagger}$ Institut für Mathematik, MA 4-5, TU Berlin, Strasse des 17 . Juni 136, D-10623 Berlin, Germany; mehrmann@math.tu-berlin.de

${ }^{\ddagger}$ This author has been supported by Deutsche Forschungsgemeinschaft through Sonderforschungsbereich 910 Control of self-organizing nonlinear systems: Theoretical methods and application concepts

${ }^{\S}$ School of Applied Mathematics and Informatics, Hanoi University of Science and Technology, 1 Dai Co Viet Str., Hanoi, Vietnam, email: ducthuank7@gmail.com.

『This author has been supported by IMU Berlin Einstein Foundation Program (EFP)
} 


\section{Introduction}

In this paper we present the stability analysis of homogeneous linear timeinvariant delay differential-algebraic equations (DDAEs) of the form

$$
E \dot{x}(t)=A x(t)+D x(t-\tau),
$$

where $E, A, D \in \mathbb{K}^{n, n}, \mathbb{K}=\mathbb{R}$ or $\mathbb{K}=\mathbb{C}$, and $\tau>0$ represents a time-delay. We study initial value problems with an initial function $\phi$, so that

$$
x(t)=\phi(t), \text { for }-\tau \leq t \leq 0 .
$$

While standard differential-algebraic equations (DAEs) without delay are today standard mathematical models for dynamical systems in many application areas, such as multibody systems, electrical circuit simulation, control theory, fluid dynamics, chemical engineering, see, e.g., 11, 4, 19, 25, 27, 33, the delay version is typically needed to model effects that do not arise instantaneously, see, e.g., [3, 16, 42]. Note that, (1) is a special case of more general neutral delay DAEs

$$
E \dot{x}(t)+F \dot{x}(t-\tau)=A x(t)+D x(t-\tau) .
$$

However, by introducing a new variable, (3) can be rewritten into the form (1) with double dimension, see [10]. For this reason here we only consider (1).

The stability and robust stability analysis for DAEs is quite different from that of ordinary differential equations (ODEs), see, e.g., [23], and has recently received a lot of attention, see, e.g., [5, 6, 12, 26, 29, 32, 37, 38] and [11] for a recent survey. In contrast to this, the stability and robust stability analysis for ordinary differential equations with delay (DDEs) is already well established, see, e.g., [20, 21, 22, 24, 35].

As an extension of both these theories, in this paper, we discuss delay differential-algebraic equations (DDAEs). Such equations, containing both algebraic constraints and delays arise, in particular, in the context of feedback control of DAE systems (where the feedback does not act instantaneously) or as limiting case for singularly perturbed ordinary delay systems, see e.g. [1, 2, 7, 8, 10, 31, 34, 43]. In sharp contrast to the situation for DDEs and DAEs even the existence and uniqueness theory of DDAEs is much less well established, see [17, 18, for a recent analysis and the discussion of many of the difficulties. This unsatisfactory situation is even more pronounced in the context of (robust) stability analysis for DDAEs. Most of the existing results are only for linear time-invariant regular DDAEs [13, 41] 
or DDAEs of special form [1, 30, 44]. Many of the results that are known for DDEs do not carry over to the DDAE case. Even the well-known spectral analysis for the exponential stability or the asymptotic stability of linear time-invariant DDAEs (1) is much more complex than that for DAEs and DDEs, see [10, 39, 43] for some special cases.

The stability analysis is usually based on the eigenvalues of the nonlinear function

$$
H(s)=s E-A-e^{-s \tau} D,
$$

associated with the Laplace transform of (1), i.e., the roots of the characteristic function

$$
p_{H}(s):=\operatorname{det} H(s) .
$$

Let us define the spectral set $\sigma(H)=\left\{s: p_{H}(s)=0\right\}$ and the spectral abscissa $\alpha(H)=\sup \left\{\operatorname{Re} s: p_{H}(s)=0\right\}$. For linear time-invariant DDEs, i.e., if $E=I_{n}$, the exponential stability is equivalent to $\alpha(H)<0$, see [20] and the spectral set $\sigma(H)$ is bounded from the right. However, for linear time-invariant DDAEs, the spectral set $\sigma(H)$ may not be bounded on the right as the following example shows.

Example 1.1. Consider the DDAE from [9]

$$
\left[\begin{array}{ll}
0 & 1 \\
0 & 0
\end{array}\right] \dot{x}(t)=\left[\begin{array}{cc}
1 & 0 \\
0 & -1
\end{array}\right] x(t)+\left[\begin{array}{ll}
0 & 0 \\
1 & 0
\end{array}\right] x(t-1),
$$

with

$$
H(s)=\left[\begin{array}{cc}
-1 & s \\
-e^{-s} & 1
\end{array}\right], p_{H}(s)=-1+s e^{-s},
$$

and thus there exist infinitely many solutions of $p_{H}(s)=0$ and their real part can be arbitrarily large, i.e., $\alpha(H)=\infty$.

The dynamics of this system is easily analyzed. Obtaining $x_{2}$ from the second equation and substituting the result into the first equation, we obtain the delay ODE $\dot{x}_{1}(t-1)=x_{1}(t)$, which is of advanced type. Thus, $x_{1}(t)=x_{1}^{(m)}(t-m)$ for $m-1 \leq t<m, m \in \mathbb{N}$. Therefore, the solution is discontinuous in general and cannot be extended on $[0, \infty)$ unless the initial function is infinitely often differentiable.

In some special cases, [31, 40, it has been shown that the exponential stability of DDAEs is equivalent to the spectral condition that $\alpha(H)<0$. In general, however this spectral condition is only necessary, but not sufficient, as the following example shows. 
Example 1.2. Consider equation (1) with

$$
E=\left[\begin{array}{llll}
1 & 0 & 0 & 0 \\
0 & 0 & 1 & 0 \\
0 & 0 & 0 & 1 \\
0 & 0 & 0 & 0
\end{array}\right], \quad A=\left[\begin{array}{cccc}
-1 & 0 & 0 & 0 \\
0 & 1 & 0 & 0 \\
0 & 0 & 1 & 0 \\
0 & 0 & 0 & 1
\end{array}\right], \quad D=\left[\begin{array}{cccc}
0 & 0 & 1 & 1 \\
0 & 0 & 0 & 0 \\
0 & 0 & 0 & 0 \\
-1 / 2 & 0 & 0 & 0
\end{array}\right]
$$

and

$$
H(s)=s E-A-e^{-s \tau} D=\left[\begin{array}{cccc}
1+s & 0 & -e^{-s \tau} & -e^{-s \tau} \\
0 & -1 & s & 0 \\
0 & 0 & -1 & s \\
e^{-s \tau} / 2 & 0 & 0 & -1
\end{array}\right] .
$$

Therefore, $p_{H}(s)=\operatorname{det} H(s)=-(1+s)\left(1-e^{-2 s \tau} / 2\right)$, the eigenvalues are $s=-1$ and $s=(-\ln 2+2 k \pi i) / 2 \tau, k \in \mathbb{Z}$, and hence all eigenvalues are in the open left half complex plane, which would suggest the exponential stability of the system, i.e., that all nontrivial solutions would be exponentially decaying. However, we will see that the asymptotic behavior (and even the existence) of the solutions depend strongly on the smoothness and the behavior of the initial function $\phi$.

Setting $x=\left[x_{1}, x_{2}, x_{3}, x_{4}\right]^{T}$, the system reads

$$
\begin{aligned}
\dot{x}_{1}(t) & =-x_{1}(t)+x_{3}(t-\tau)+x_{4}(t-\tau), \\
\dot{x}_{3}(t) & =x_{2}(t) \\
\dot{x}_{4}(t) & =x_{3}(t) \\
0 & =x_{4}(t)-x_{1}(t-\tau) / 2 .
\end{aligned}
$$

Solving for $x_{4}$ in the last equation and substituting this and $x_{3}$ obtained from the third equation into the first equation, we arrive at

$$
\dot{x}_{1}(t)=-x_{1}(t)+\dot{x}_{1}(t-2 \tau) / 2+x_{1}(t-2 \tau) / 2 .
$$

This underlying neutral delay ODE has the characteristic function $-p_{H}(s)$, so its spectral set is the same as that of the original system. The spectral condition ensures the exponential stability of the underlying equation for $x_{1}$, see [20]. However, $x_{2}$ and $x_{3}$ are just the second and the first derivatives of $x_{4}(t)=x_{1}(t-\tau) / 2$. Thus, if the first component of $\phi$ is not differentiable on $(-\tau, 0)$ or it is differentiable (almost everywhere) but the derivative is unbounded, then the solution does not exist or is unbounded. For example, the function $\phi_{1}(t)=t \sin (1 / t)$ is continuous on $[-\tau, 0]$, differentiable on $(-\tau, 0)$, but the derivative is obviously unbounded. 
Example 1.2 shows that linear time-invariant DDAEs may not be exponentially stable although all roots of the characteristic function are in the open left half complex plane. To characterize when the roots of the characteristic function allow the classification of stability, in this paper we derive necessary and sufficient conditions that guarantee that for time-invariant DDAEs exponential stability is equivalent to the condition that all eigenvalues of $H$ have negative real part and thus extend recent results of [31].

With a characterization of exponential stability at hand we also study the question of robust stability for linear time-invariant DDAEs, i.e., we discuss the structured stability radius of maximal perturbations that are allowed to the coefficients so that the system keeps its exponential stability. These results extend previous results on DDEs and DAEs in [5, 6, 12, 11, 24, 35.

The paper is organized as follows. In the next section we introduce the basic notation and present some preliminary results. Then, in Section 3 , we characterize exponential stability for general linear time-invariant DDAEs. In Section 44, we will introduce allowable perturbations for two different classes of systems (1) and present a formula for the structured stability radius for DDAEs. In Section 5, some conclusions and open problems close the paper.

\section{Preliminaries}

In the following, we denote by $I_{n} \in \mathbb{C}^{n, n}$ the identity matrix, by $0 \in \mathbb{C}^{n, n}$ the zero matrix, by $A C\left(\mathbb{I}, \mathbb{C}^{n}\right)$ the space of absolutely continuous functions, and by $C_{p w}^{k}\left(\mathbb{I}, \mathbb{C}^{n}\right)$ the space of $k$-times piecewise continuously differentiable functions from $\mathbb{I} \subset[0, \infty)$ to $\mathbb{C}^{n}$.

Definition 2.1. A function $x(\cdot, \phi):[0, \infty) \rightarrow \mathbb{C}^{n}$ is called solution of the initial value problem (1) -(2), if $x \in A C\left([0, \infty), \mathbb{C}^{n}\right)$ and $x(\cdot, \phi)$ satisfies (1) almost everywhere. An initial function $\phi$ is called consistent with (1) if the associated initial value problem (1) has at least one solution.

System (1) is called solvable if for every consistent initial function $\phi$, the associated initial value problem (1)-(2) has a solution. It is called regular if it is solvable and the solution is unique.

Note that instead of seeking solutions in $A C\left([0, \infty), \mathbb{C}^{n}\right)$, alternatively we often consider the space $C_{p w}^{1}\left([0, \infty), \mathbb{C}^{n}\right)$. In fact, equation (1) may not be satisfied at (countably many) points, which usually arise at multiples of the delay time $\tau$. 
Definition 2.2. System (1)-(2) is called exponentially stable if there exist constants $K>0, \omega>0$ such that

$$
\|x(t, \phi)\| \leq K e^{-\omega t}\|\phi\|_{\infty}
$$

for all $t \geq 0$ and all consistent initial functions $\phi$, where $\|\phi\|_{\infty}=\sup _{-\tau \geq t \geq 0}\|\phi(t)\|$.

Note that one can transform (1) in such a way that a given solution $x(t ; \phi)$ is mapped to the trivial solution by simply shifting the arguments.

Definition 2.3. A matrix pair $(E, A), E, A \in \mathbb{C}^{n, n}$ is called regular if there exists $s \in \mathbb{C}$ such that $\operatorname{det}(s E-A)$ is different from zero. Otherwise, if $\operatorname{det}(s E-A)=0$ for all $s \in \mathbb{C}$, then we say that $(E, A)$ is singular.

If $(E, A)$ is regular, then a complex number $\lambda$ is called a (generalized finite) eigenvalue of $(E, A)$ if $\operatorname{det}(\lambda E-A)=0$. The set of all (finite) eigenvalues of $(E, A)$ is called the (finite) spectrum of the pencil $(E, A)$ and denoted by $\sigma(E, A)$. If $E$ is singular and the pair is regular, then we say that $(E, A)$ has the eigenvalue $\infty$.

Regular pairs $(E, A)$ can be transformed to Weierstraß-Kronecker canonical form, see [4, 14, 15], i.e., there exist nonsingular matrices $W, T \in \mathbb{C}^{n, n}$ such that

$$
E=W\left[\begin{array}{cc}
I_{r} & 0 \\
0 & N
\end{array}\right] T^{-1}, A=W\left[\begin{array}{cc}
J & 0 \\
0 & I_{n-r}
\end{array}\right] T^{-1},
$$

where $I_{r}, I_{n-r}$ are identity matrices of indicated size, $J \in \mathbb{C}^{r, r}$, and $N \in$ $\mathbb{C}^{(n-r),(n-r)}$ are matrices in Jordan canonical form and $N$ is nilpotent. If $E$ is invertible, then $r=n$, i.e., the second diagonal block does not occur.

Definition 2.4. Consider a regular pair $(E, A)$ with $E, A \in \mathbb{C}^{n, n}$ in WeierstraßKronecker form (7). If $r<n$ and $N$ has nilpotency index $\nu \in\{1,2, \ldots\}$, i.e., $N^{\nu}=0, N^{i} \neq 0$ for $i=1,2, \ldots, \nu-1$, then $\nu$ is called the index of the pair $(E, A)$ and we write $\operatorname{ind}(E, A)=\nu$. If $r=n$ then the pair has index $\nu=0$.

For system (1) with a regular pair $(E, A)$, the existence and uniqueness of solutions has been studied in [7, 8, 9], and for the general case in [17]. It follows from Corollary 4.12 in [17] that (1)-22) has a unique solution if and only if the initial condition $\phi$ is consistent and $p_{H}(s)=\operatorname{det}(H(s)) \not \equiv 0$.

For a matrix triple $(E, A, D) \in \mathbb{C}^{n, n} \times \mathbb{C}^{n, n} \times \mathbb{C}^{n, n}$, there always exists a nonsingular matrix $W \in \mathbb{C}^{n, n}$ such that

$$
W^{-1} E=\left[\begin{array}{c}
E_{1} \\
0 \\
0
\end{array}\right], \quad W^{-1} A=\left[\begin{array}{c}
A_{1} \\
A_{2} \\
0
\end{array}\right], \quad W^{-1} D=\left[\begin{array}{c}
D_{1} \\
D_{2} \\
D_{3}
\end{array}\right],
$$


where $E_{1}, A_{1}, D_{1} \in \mathbb{C}^{d, n}, A_{2}, D_{2} \in \mathbb{C}^{a, n}, D_{3} \in \mathbb{C}^{h, n}$ with $d+a+h=n$, rank $E_{1}=\operatorname{rank} E=d$, and rank $A_{2}=a$. Then, system (1) can be scaled by $W^{-1}$ to obtain

$$
\begin{aligned}
E_{1} \dot{x}(t) & =A_{1} x(t)+D_{1} x(t-\tau), \\
0 & =A_{2} x(t)+D_{2} x(t-\tau), \\
0 & =D_{3} x(t-\tau) .
\end{aligned}
$$

In practice, the scaling matrix $W$ and the transformed coefficient matrices can be easily constructed as follows. Let $U$ be the left unitary factor of the singular value decomposition (SVD) of $E$, i.e., $U$ consists of the left singular vectors of $E$. Assuming that $\operatorname{rank} E=d$, we decompose $U=\left[U_{1}, U_{2}\right]$ accordingly. Then let $\tilde{U}=\left[\tilde{U}_{2}, \tilde{U}_{3}\right]$ be the left unitary factor of the SVD of $U_{2}^{*} A$ with $\operatorname{rank} U_{2}^{*} A=a$. Then, we define $W=U \operatorname{diag}\left(I_{d}, \tilde{U}\right)$. It is easy to check that multiplying by $W^{-1}=\operatorname{diag}\left(I_{d}, \tilde{U}^{*}\right) U^{*}$, the form $(8)$ is obtained with

$E_{1}=U_{1}^{*} E, A_{1}=U_{1}^{*} A, D_{1}=U_{1}^{*} D, A_{2}=\tilde{U}_{2}^{*} U_{2}^{*} A, D_{2}=\tilde{U}_{2}^{*} U_{2}^{*} D, D_{3}=\tilde{U}_{3}^{*} U_{2}^{*} D$.

We immediately see that to obtain solvability of the equation, the initial function has to be in the set

$\mathcal{S}:=\left\{\phi: \phi \in A C\left([-\tau, 0], \mathbb{C}^{n}\right), A_{2} \phi(0)+D_{2} \phi(-\tau)=0, D_{3} \phi(t)=0\right.$ for all $\left.t \in[-\tau, 0]\right\}$

Shifting the time in the last equation of $(9)$ by $\tau$, we obtain

$$
\begin{aligned}
E_{1} \dot{x}(t) & =A_{1} x(t)+D_{1} x(t-\tau), \\
A_{2} x(t) & =-D_{2} x(t-\tau), \\
0 & =D_{3} x(t) .
\end{aligned}
$$

Differentiating the second and third equation of $(10)$, we get

$$
\begin{aligned}
& E_{1} \dot{x}(t)=A_{1} x(t)+D_{1} x(t-\tau), \\
& A_{2} \dot{x}(t)=-D_{2} \dot{x}(t-\tau), \\
& D_{3} \dot{x}(t)=0
\end{aligned}
$$

Following the concept of strangeness-index in [25] we make the following definition, see also [17]. 
Definition 2.5. Equation (1) is called strangeness-free if there exists a nonsingular matrix $W \in \mathbb{C}^{n, n}$ that transforms the triple $(E, A, D)$ to the form (8) and

$$
\operatorname{rank}\left[\begin{array}{c}
E_{1} \\
A_{2} \\
D_{3}
\end{array}\right]=n
$$

It is easy to show that the strangeness-free property is invariant with respect to the choice of $W$. If (1) is strangeness-free then, setting

$$
\widehat{E}=\left[\begin{array}{c}
E_{1} \\
A_{2} \\
D_{3}
\end{array}\right], \widehat{A}=\left[\begin{array}{c}
A_{1} \\
0 \\
0
\end{array}\right], \widehat{D}=\left[\begin{array}{c}
D_{1} \\
0 \\
0
\end{array}\right], \widehat{F}=\left[\begin{array}{c}
0 \\
-D_{2} \\
0
\end{array}\right],
$$

the implicit system is (11) is equivalent to the neutral linear time-invariant DDE

$$
\dot{x}(t)=\widehat{E}^{-1} \widehat{A} x(t)+\widehat{E}^{-1} \widehat{D} x(t-\tau)+\widehat{E}^{-1} \widehat{F} \dot{x}(t-\tau),
$$

which admits a unique solution that satisfies the consistent initial condition (2).

In the next section we present necessary and sufficient conditions such that the exponential stability for linear time-invariant DDAEs is characterized by the spectral function.

\section{$3 \quad$ Exponential stability of linear DDAEs}

In this section we show that for strangeness-free systems the spectral condition characterizes exponential stability.

Theorem 3.1. Suppose that equation (1) is strangeness-free. Then equation (1) is exponentially stable if and only if $\alpha(H)<0$.

Proof. Necessity. Suppose that equation (1) is exponentially stable, i.e., inequality (6) holds with positive constants $K$ and $\omega$, but $\alpha(H) \geq 0$. Then there exists an eigenvalue $\lambda \in \sigma(H)$ with $\operatorname{Re} \lambda>-\omega$. Let $v \neq 0$ be an eigenvector associated with $\lambda$, i.e., $\left(\lambda E-A-e^{-\lambda \tau} D\right) v=0$, then obviously $x(t)=e^{\lambda t} v$ is a solution of equation (1), but it does not satisfy (6). This is a contradiction and thus $\alpha(H)<0$.

Sufficiency. Suppose that $\alpha(H)<0$ and consider a solution $x$ of (1). As seen in the previous section, $x$ also satisfies the neutral delay ODE system 
(12), whose spectral function is

$$
\begin{aligned}
\widehat{H}(s) & =s I-\widehat{E}^{-1} \widehat{A}-e^{-s \tau} \widehat{E}^{-1} \widehat{D}-s e^{-s \tau} \widehat{E}^{-1} \widehat{F} \\
& =\widehat{E}^{-1}\left(s \widehat{E}-\widehat{A}-e^{-s \tau} \widehat{D}-s e^{-s \tau} \widehat{F}\right) .
\end{aligned}
$$

It is easy to see that $\sigma(\widehat{H})=\sigma(H) \cup\{0\}$. We have $\alpha(\widehat{H})=0$, but because $\alpha(H)<0,0$ is an isolated (and semisimple) eigenvalue. It has been shown in [20, Chapter 12] that the solutions of $(12)$ can be represented in the form

$$
x(t)=\widehat{v}+\widehat{x}^{*}(t),
$$

where $\widehat{x}^{*}(t)$ satisfies (6) and either $\widehat{v}=0$ or $\widehat{v}$ is an eigenvector associated with the eigenvalue $\lambda=0$ of $\hat{H}(\lambda)$. Hence, we have

$$
A_{1} \widehat{v}+D_{1} \widehat{v}=0 .
$$

Moreover, since $\lim _{t \rightarrow \infty} \widehat{x}^{*}(t)=0$, from the second and the third equations of 10 , it follows that

$$
A_{2} \widehat{v}+D_{2} \widehat{v}=D_{3} \widehat{v}=0 .
$$

From (14) and (15), it follows that $H(0) \widehat{v}=0$. But since $0 \notin \sigma(H)$, this implies that $\widehat{v}=0$ and hence $x(t)=\widehat{x}^{*}(t)$. Thus, equation (1) is exponentially stable.

Remark 3.2. In the proof of Theorem 3.1, we see that $\alpha(H) \leq \alpha(\widehat{H})$ always holds. Thus, if system (1) is strangeness-free then the spectral set $\sigma(H)$ is bounded from the right, or equivalently the spectral abscissa satisfies $\alpha(H)<\infty$.

Now we consider the case when the pair $(E, A)$ (1) is regular and it is transformed into the Weierstraß-Kronecker canonical form (7). Setting

$$
W^{-1} D T=\left[\begin{array}{ll}
D_{11} & D_{12} \\
D_{21} & D_{22}
\end{array}\right], T^{-1} x(t)=\left[\begin{array}{l}
x_{1}(t) \\
x_{2}(t)
\end{array}\right], T^{-1} \phi(t)=\left[\begin{array}{l}
\phi_{1}(t) \\
\phi_{2}(t)
\end{array}\right],
$$

with $D_{11} \in \mathbb{C}^{r, r}, D_{12} \in \mathbb{C}^{r, n-r}, D_{21} \in \mathbb{C}^{n-r, r}, D_{22} \in \mathbb{C}^{n-r, n-r}$, and $x_{1}, x_{2}, \phi_{1}, \phi_{2}$ partitioned analogously. Then equation (1) is equivalent to the system

$$
\begin{aligned}
\dot{x}_{1}(t) & =A_{11} x_{1}(t)+D_{11} x_{1}(t-\tau)+D_{12} x_{2}(t-\tau), \\
N \dot{x}_{2}(t) & =x_{2}(t)+D_{21} x_{1}(t-\tau)+D_{22} x_{2}(t-\tau),
\end{aligned}
$$


with initial conditions

$$
x_{i}(t)=\phi_{i}(t), \text { for } t \in[-\tau, 0], i=1,2 .
$$

From the explicit solution formula for linear time-invariant DAEs, see [7, 25], the second equation of (17) implies that

$x_{2}(t)=-D_{21} x_{1}(t-\tau)-D_{22} x_{2}(t-\tau)-\sum_{i=1}^{\nu-1}\left(N^{i} D_{21} x_{1}^{(i)}(t-\tau)+N^{i} D_{22} x_{2}^{(i)}(t-\tau)\right)$,

and for $t \in[0, \tau)$, we get

$$
x_{2}(t)=-D_{21} \phi_{1}(t)-D_{22} \phi_{2}(t)-\sum_{i=1}^{\nu-1}\left(N^{i} D_{21} \phi_{1}^{(i)}(t)+N^{i} D_{22} \phi_{2}^{(i)}(t)\right) .
$$

It follows that $\phi$ needs to be differentiable at least $\nu$ times if the coefficients $D_{21}$ and $D_{22}$ do not satisfy further conditions. Extending this argument to $t \in[\tau, 2 \tau),[2 \tau, 3 \tau)$, etc., the solution cannot be extended to the full real half-line unless the initial function $\phi$ is infinitely often differentiable or the coefficient associated with the delay is highly structured.

Corollary 3.3. Consider the DDAE (1)-(2) with a regular pair $(E, A)$, $\operatorname{ind}(E, A) \leq 1$, and its associated spectral function $H$. Then equation (1) is exponentially stable if and only if $\alpha(H)<0$.

Proof. If $\operatorname{ind}(E, A) \leq 1$ then the system is obviously strangeness-free in the sense of Definition 2.5 with $d+a=n$ and $h=0$. Thus, by Theorem 3.1. the system is exponentially stable if and only if $\alpha(H)<0$.

We note that the result of Corollary 3.3 is obtained in [31] by a direct proof.

Let us now consider exponential stability for the case that ind $(E, A)>1$ or for the case that $(E, A)$ is singular.

In order to avoid an infinite number differentiations of $\phi$ induced by (20), it is reasonable to assume that for a system in WeierstraßKronecker form (7) with transformed matrices as in (16) the allowable delay condition $N D_{2 i}=0, i=1,2$ holds. Note that this condition is trivially true for the index-1 case, since then we have $N=0$. In terms of the original coefficients for (1) for a regular pair $(E, A)$ with arbitrary index this allowable delay condition can be described as follows.

Choose any fixed $\hat{s} \in \mathbb{C}$ such that $\operatorname{det}(\hat{s} E-A) \neq 0$ and set

$$
\hat{E}=(\hat{s} E-A)^{-1} E, \quad \hat{D}=(\hat{s} E-A)^{-1} D .
$$


Proposition 3.4. Consider a DDAE of the form (1) with a regular pair $(E, A)$ of arbitrary index, let $\hat{s} \in \mathbb{C}$ be such that $\operatorname{det}(s E-A) \neq 0$, and consider the system (7) after transformation to Weierstraß-Kronecker canonical form. Then the allowable delay conditions $N D_{21}=0$ and $N D_{22}=0$ are simultaneously satisfied if and only if

$$
\left(I-\hat{E}^{D} \hat{E}\right) \hat{E} \hat{D}=0,
$$

where $\hat{E}^{D}$ denotes the Drazin inverse of $\hat{E}$.

Proof. From (7) it follows that

$$
\hat{E}=T\left[\begin{array}{cc}
\left(\hat{s} I_{r}-J\right)^{-1} & 0 \\
0 & \left(\hat{s} N-I_{n-r}\right)^{-1} N
\end{array}\right] T^{-1},
$$

and

$$
\hat{D}=T\left[\begin{array}{cc}
\left(\hat{s} I_{r}-J\right)^{-1} D_{11} & \left(\hat{s} I_{r}-J\right)^{-1} D_{12} \\
\left(\hat{s} N-I_{n-r}\right)^{-1} D_{21} & \left(\hat{s} N-I_{n-r}\right)^{-1} D_{22}
\end{array}\right] T^{-1} .
$$

Therefore,

$$
\hat{E}^{D}=T\left[\begin{array}{cc}
\left(\hat{s} I_{r}-J\right)^{-1} & 0 \\
0 & 0
\end{array}\right] T^{-1}
$$

and by elementary calculations we get

$$
\left(I-\hat{E}^{D} \hat{E}\right) \hat{E} \hat{D}=T\left[\begin{array}{cc}
0 & 0 \\
\left(\hat{s} N-I_{n-r}\right)^{-2} N D_{21} & \left(\hat{s} N-I_{n-r}\right)^{-2} N D_{22}
\end{array}\right] T^{-1} .
$$

Thus we have that $\left(I-\hat{E}^{D} \hat{E}\right) \hat{E} \hat{D}=0$ if and only if $N D_{21}=0$ and $N D_{22}=$ 0 .

Using proposition 3.4, we have the following characterization of exponential stability for DDAEs with regular pair $(E, A)$.

Theorem 3.5. Consider the DDAE (1)-(2) with a regular pair $(E, A)$ satisfying (22). Then equation (1) is exponentially stable if and only if $\alpha(H)<0$.

Proof. Necessity. The proof is analogous to that of Theorem 3.1 and we conclude that if equation (1) is exponentially stable then $\alpha(H)<0$.

Sufficiency. Suppose that $\alpha(H)<0$. Since the pair $(E, A)$ is regular, it follows that (1) $-(2)$ is equivalent to system in canonical form (17). Under the assumption (22), we have $N D_{2 i}=0, i=1,2$, and thus 19 is reduced to

$$
0=x_{2}(t)+D_{21} x_{1}(t-\tau)+D_{22} x_{2}(t-\tau) \text {. }
$$


This implies that $\left[\begin{array}{ll}x_{1}^{T} & x_{2}^{T}\end{array}\right]^{T}$ is also a solution to the index-1 DDAE

$$
\left[\begin{array}{cc}
I_{r} & 0 \\
0 & 0
\end{array}\right]\left[\begin{array}{l}
\dot{x}_{1}(t) \\
\dot{x}_{2}(t)
\end{array}\right]=\left[\begin{array}{cc}
A_{11} & 0 \\
0 & I_{n-r}
\end{array}\right]\left[\begin{array}{l}
x_{1}(t) \\
x_{2}(t)
\end{array}\right]+\left[\begin{array}{cc}
D_{11} & D_{12} \\
D_{21} & D_{22}
\end{array}\right]\left[\begin{array}{l}
x_{1}(t-\tau) \\
x_{2}(t-\tau)
\end{array}\right],
$$

with the characteristic function

$$
\tilde{H}(s)=s\left[\begin{array}{cc}
I_{r} & 0 \\
0 & 0
\end{array}\right]-\left[\begin{array}{cc}
s I_{r}-A_{11} & 0 \\
0 & I_{n-r}
\end{array}\right]-e^{-s \tau}\left[\begin{array}{cc}
D_{11} & D_{12} \\
D_{21} & D_{22}
\end{array}\right] .
$$

Using the Weierstraß-Kronecker canonical form (7), we have that

$$
W^{-1} H(s) T=\left[\begin{array}{cc}
s I_{r}-A_{11} & 0 \\
0 & s N-I_{n-r}
\end{array}\right]-e^{-s \tau}\left[\begin{array}{cc}
D_{11} & D_{12} \\
D_{21} & D_{22}
\end{array}\right] .
$$

Since $N D_{2 i}=0, i=1,2$, and $-\left(s N-I_{n-r}\right)^{-1}=\sum_{i=0}^{\nu-1}(s N)^{i}$, it follows that

$$
\begin{aligned}
& {\left[\begin{array}{cc}
I_{r} & 0 \\
0 & -\left(s N-I_{n-r}\right)^{-1}
\end{array}\right] W^{-1} H(s) T } \\
= & {\left[\begin{array}{cc}
s I_{r}-A_{11} & 0 \\
0 & -I_{n-r}
\end{array}\right]-e^{-s \tau}\left[\begin{array}{cc}
D_{11} & D_{12} \\
-\left(s N-I_{n-r}\right)^{-1} D_{21} & -\left(s N-I_{n-r}\right)^{-1} D_{22}
\end{array}\right] } \\
= & {\left[\begin{array}{cc}
s I_{r}-A_{11} & 0 \\
0 & -I_{n-r}
\end{array}\right]-e^{-s \tau}\left[\begin{array}{cc}
D_{11} & D_{12} \\
\sum_{i=0}^{\nu}(s N)^{i} D_{21} & \sum_{i=0}^{\nu-1}(s N)^{i} D_{22}
\end{array}\right] } \\
= & {\left[\begin{array}{cc}
s I_{r}-A_{11} & 0 \\
0 & -I_{m-r}
\end{array}\right]-e^{-s \tau}\left[\begin{array}{ll}
D_{11} & D_{12} \\
D_{21} & D_{22}
\end{array}\right] } \\
= & \tilde{H}(s) .
\end{aligned}
$$

This implies that $\operatorname{det} \tilde{H}(s)=0$ if and only if $\operatorname{det} H(s)=0$, and hence $\alpha(\tilde{H})=\alpha(H)<0$. Thus, by Corollary 3.3, system $24-18$ is exponentially stable and hence system (1)-(2) is exponentially stable.

For the system in Example 1.2 which has a regular pair $(E, A)$ that is already in Weierstraß-Kronecker form, we have $N D_{21} \neq 0$ but $N D_{22}=0$ and the system has $\alpha(H)<0$ but the system is not exponentially stable. The following example presents the same observation for the case $N D_{21}=0$ but $N D_{22} \neq 0$. 
Example 3.6. Consider equation (1) with

$$
E=\left[\begin{array}{llll}
1 & 0 & 0 & 0 \\
0 & 0 & 1 & 0 \\
0 & 0 & 0 & 1 \\
0 & 0 & 0 & 0
\end{array}\right], \quad A=\left[\begin{array}{cccc}
-1 & 0 & 0 & 0 \\
0 & 2 & 0 & 0 \\
0 & 0 & 2 & 0 \\
0 & 0 & 0 & 2
\end{array}\right], \quad D=\left[\begin{array}{llll}
0 & 0 & 0 & 1 \\
0 & 1 & 0 & 0 \\
0 & 0 & 1 & 0 \\
0 & 0 & 0 & 1
\end{array}\right]
$$

We then have

$$
H(s)=s E-A-e^{-s \tau} D=\left[\begin{array}{cccc}
1+s & 0 & 0 & -e^{-s \tau} \\
0 & -2-e^{-s \tau} & s & 0 \\
0 & 0 & -2-e^{-s \tau} & s \\
0 & 0 & 0 & -2-e^{-s \tau}
\end{array}\right] .
$$

Therefore, $\operatorname{det} H(s)=-(1+s)\left(2+e^{-s \tau}\right)^{3}$, the eigenvalues are $\lambda=-1$ and $s=(-\ln 2+(2 k+1) \pi) / \tau, k \in \mathbb{Z}$, and hence all eigenvalues are in the open left half complex plane.

However, the system can be written as

$$
\begin{aligned}
\dot{x}_{1}(t) & =-x_{1}(t)+x_{4}(t-\tau), \\
\dot{x}_{3}(t) & =2 x_{2}(t)+x_{2}(t-\tau), \\
\dot{x}_{4}(t) & =2 x_{3}(t)+x_{3}(t-\tau), \\
0 & =2 x_{4}(t)+x_{4}(t-\tau) .
\end{aligned}
$$

It is clear that if $\phi_{4}$ is not sufficiently smooth or its derivatives are unbounded, then the second and the third component solutions cannot be extended or they are unbounded. If the solution is defined for all $t \geq 0$, it depends on the derivatives of the initial function in general. Thus, the system is not exponentially stable.

We have seen that the spectral condition $\alpha(H)<0$ is necessary for the exponential stability of (1), but in general it is not sufficient. Introducing further restrictions on the delays, we get that exponential stability is equivalent to the spectral condition.

\section{Robust exponential stability}

We have seen in the previous section that under some extra conditions the exponential stability of a linear time-invariant DDAE can be characterized by the spectral properties of the matrix function $H(s)$. Typically, however, the coefficient functions are not exactly known, since they arise, e.g., from a modeling, or system identification process, or as coefficient matrices from 
a discretization process. Thus, a more realistic scenario for the stability analysis is to analyze the robustness of the exponential stability under small perturbations. To perform this analysis, in this section we study the behavior of the spectrum of the triple of coefficient matrices $(E, A, D)$ under structured perturbations in the matrices $E, A, D$.

Suppose that system (1) is exponentially stable and consider a perturbed system

$$
\left(E+B_{1} \Delta_{1} C\right) \dot{x}(t)=\left(A+B_{2} \Delta_{2} C\right) x(t)+\left(D+B_{3} \Delta_{3} C\right) x(t-\tau),
$$

where $\Delta_{i} \in \mathbb{C}^{p_{i}, q}, i=1,2,3$ are perturbations and $B_{i} \in \mathbb{C}^{n, p_{i}}, i=1,2,3$, $C \in \mathbb{C}^{q, n}$, are matrices that restrict the structure of the perturbations. We could also consider different matrices $C_{i}$ in each of the coefficients but for simplicity we assume that the column structure in the perturbations is the same for all coefficients. Set

$$
\Delta=\left[\begin{array}{c}
\Delta_{1} \\
\Delta_{2} \\
\Delta_{3}
\end{array}\right], B=\left[\begin{array}{lll}
B_{1} & B_{2} & B_{3}
\end{array}\right],
$$

and $p=p_{1}+p_{2}+p_{3}$ and consider the set of destabilizing perturbations

$$
\mathcal{V}_{\mathbb{C}}(E, A, D ; B, C)=\left\{\Delta \in \mathbb{C}^{p \times q}: 25 \text { is not exponentially stable }\right\} .
$$

Then we define the structured complex stability radius of (1) subject to structured perturbations as in 25 as

$$
r_{\mathbb{C}}(E, A, D ; B, C)=\inf \left\{\|\Delta\|: \Delta \in \mathcal{V}_{\mathbb{C}}(E, A, D ; B, C)\right\},
$$

where $\|\cdot\|$ is a matrix norm induced by a vector norm. If only real perturbations $\Delta$ are considered, then we use the term structured real stability radius but here we focus on the complex stability radius.

With $H$ as in (4), we introduce the transfer functions

$G_{1}(\lambda)=-\lambda C H(\lambda)^{-1} B_{1}, G_{2}(\lambda)=C H(\lambda)^{-1} B_{2}, G_{3}(\lambda)=e^{-\lambda \tau} C H(\lambda)^{-1} B_{3}$, and with

$$
G(\lambda)=\left[\begin{array}{lll}
G_{1}(\lambda) & G_{2}(\lambda & G_{3}(\lambda)
\end{array}\right],
$$

we obtain an explicit formula for the structured stability radius.

Theorem 4.1. Suppose that system (1) is exponentially stable. Then the structured stability radius of (1) subject to structured perturbations as in (25) satisfies the inequality

$$
r_{\mathbb{C}}(E, A, D ; B, C) \leq\left(\sup _{\operatorname{Re} \lambda \geq 0}\|G(\lambda)\|\right)^{-1} .
$$


Proof. Let $\epsilon$ be an arbitrary positive number and let $\lambda_{0} \in \overline{\mathbb{C}}^{+}$, where $\overline{\mathbb{C}}^{+}=$ $\{\lambda \in \mathbb{C}, \operatorname{Re} \lambda \geq 0\}$ is the closed right-half plane, be such that

$$
\left\|G\left(\lambda_{0}\right)\right\|^{-1} \leq\left(\sup _{\operatorname{Re} \lambda \geq 0}\|G(\lambda)\|\right)^{-1}+\epsilon,
$$

and let $u \in \mathbb{C}^{n}$ be such that $\|u\|=1$ and

$$
\left\|G\left(\lambda_{0}\right) u\right\|=\left\|G\left(\lambda_{0}\right)\right\|
$$

Furthermore, let $y \in \mathbb{C}^{q}$ be such that $\|y\|=1$ and

$$
y^{H}\left(G\left(\lambda_{0}\right) u\right)=\left\|G\left(\lambda_{0}\right) u\right\|=\left\|G\left(\lambda_{0}\right)\right\|,
$$

and set

$$
\Delta=\left\|G\left(\lambda_{0}\right)\right\|^{-1} u y^{H}, x=H\left(\lambda_{0}\right)^{-1}\left[\begin{array}{lll}
-\lambda_{0} B_{1} & B_{2} & e^{-\lambda_{0} \tau} B_{3}
\end{array}\right] u .
$$

Then

$$
\|\Delta\| \leq\left\|G\left(\lambda_{0}\right)\right\|^{-1}\|u\|\|y\|=\left\|G\left(\lambda_{0}\right)\right\|^{-1},
$$

and

$$
\Delta G\left(\lambda_{0}\right) u=\frac{u}{\left\|G\left(\lambda_{0}\right)\right\|}\left\|G\left(\lambda_{0}\right)\right\|=u .
$$

Since $u \neq 0$, it follows that $\|\Delta\| \geq\left\|G\left(\lambda_{0}\right)\right\|^{-1}$ and thus $\|\Delta\|=\left\|G\left(\lambda_{0}\right)\right\|^{-1}$. Since

$$
G\left(\lambda_{0}\right) u=C H\left(\lambda_{0}\right)^{-1}\left[\begin{array}{lll}
-\lambda_{0} B_{1} & B_{2} & e^{-\lambda_{0} \tau} B_{3}
\end{array}\right] u \neq 0,
$$

it follows that $\left[\begin{array}{lll}-\lambda_{0} B_{1} & B_{2} & e^{-\lambda_{0} \tau} B_{3}\end{array}\right] u \neq 0$, and hence $x \neq 0$.

On the other hand, by (30) and (31) we have

$$
\begin{aligned}
H\left(\lambda_{0}\right) x & =\left[\begin{array}{lll}
-\lambda_{0} B_{1} & B_{2} & e^{-\lambda_{0} \tau} B_{3}
\end{array}\right] u=\left[\begin{array}{lll}
-\lambda_{0} B_{1} & B_{2} & e^{-\lambda_{0} \tau} B_{3}
\end{array}\right] \Delta G\left(\lambda_{0}\right) u \\
& =\left[\begin{array}{lll}
-\lambda_{0} B_{1} & B_{2} & e^{-\lambda_{0} \tau} B_{3}
\end{array}\right] \Delta C H\left(\lambda_{0}\right)^{-1}\left[\begin{array}{lll}
-\lambda_{0} B_{1} & B_{2} & e^{-\lambda_{0} \tau} B_{3}
\end{array}\right] u \\
& =\left[\begin{array}{lll}
-\lambda_{0} B_{1} & B_{2} & e^{-\lambda_{0} \tau} B_{3}
\end{array}\right] \Delta C x \\
& =\left(-\lambda_{0} B_{1} \Delta_{1} C_{1}+B_{2} \Delta_{2} C_{2}+e^{-\lambda \tau} B_{3} \Delta_{3} C_{3}\right) x,
\end{aligned}
$$

and thus,

$$
\left(\lambda_{0}\left(E+B_{1} \Delta_{1} C_{1}\right)-\left(A+B_{2} \Delta_{2} C_{2}\right)-e^{-\lambda_{0} \tau}\left(D+B_{3} \Delta_{3} C_{3}\right)\right) x=0 .
$$


This relation implies that $\lambda_{0}$ is a root of the characterestic function associated with (25). Since $\operatorname{Re} \lambda_{0} \geq 0$, it follows that (25) is not exponentially stable. Thus, $\Delta \in \mathcal{V}_{\mathbb{C}}(E, A, D ; B, C)$, which implies that

$$
r_{\mathbb{C}}(E, A, D ; B, C) \leq\|\Delta\|=\left\|G\left(\lambda_{0}\right)\right\|^{-1} \leq\left(\sup _{\operatorname{Re} \lambda \geq 0}\|G(\lambda)\|\right)^{-1}+\epsilon .
$$

Since $\epsilon$ is arbitrary, if follows that

$$
r_{\mathbb{C}}(E, A, D ; B, C) \leq\left(\sup _{\operatorname{Re} \lambda \geq 0}\|G(\lambda)\|\right)^{-1},
$$

and the proof is complete.

For every perturbation $\Delta$ as in $(26)$ we define

$$
H_{\Delta}(\lambda)=\lambda\left(E+B_{1} \Delta_{1} C\right)-\left(A+B_{2} \Delta_{2} C\right)-e^{-\lambda \tau}\left(D+B_{3} \Delta_{3} C\right)
$$

and have the following proposition.

Proposition 4.2. Consider system (1) and the perturbed system (25). If the associated spectral abscissa satisfy $\alpha(H)<0$ and $\alpha\left(H_{\Delta}\right) \geq 0$, then we have

$$
\|\Delta\| \geq\left(\sup _{\operatorname{Re} \lambda \geq 0}\|G(\lambda)\|\right)^{-1} .
$$

Proof. If $\sup _{\operatorname{Re} \lambda \geq 0}\|G(\lambda)\|=\infty$ then (33) holds trivially. Therefore, we may assume that

$$
\sup _{\operatorname{Re} \lambda \geq 0}\|G(\lambda)\|<\infty .
$$

Since $\alpha\left(H_{\Delta}\right) \geq 0$, we have two cases.

Case 1: There exists $\lambda_{0} \in \sigma\left(H_{\Delta}\right)$ such that $\operatorname{Re} \lambda_{0} \geq 0$. Then, there exists a nonzero $x \in \mathbb{C}^{n}$ such that $H_{\Delta}\left(\lambda_{0}\right) x=0$, and we have

$$
0=H_{\Delta}\left(\lambda_{0}\right) x=H\left(\lambda_{0}\right) x-\left[\begin{array}{lll}
-\lambda_{0} B_{1} & B_{2} & e^{-\lambda_{0} \tau} B_{3}
\end{array}\right] \Delta C x .
$$

Since $H\left(\lambda_{0}\right)$ is invertible, we have that $H\left(\lambda_{0}\right) x \neq 0$ and thus

$$
x=H\left(\lambda_{0}\right)^{-1}\left[\begin{array}{lll}
-\lambda_{0} B_{1} & B_{2} & e^{-\lambda_{0} \tau} B_{3}
\end{array}\right] \Delta C x,
$$

and also $C x \neq 0$. By multiplying $C$ from the left on both sides of (34), we obtain

$$
C x=C H\left(\lambda_{0}\right)^{-1}\left[\begin{array}{lll}
-\lambda_{0} B_{1} & B_{2} & e^{-\lambda_{0} \tau} B_{3}
\end{array}\right] \Delta C x=G\left(\lambda_{0}\right) \Delta C x,
$$


and hence,

$$
\|C x\| \leq\left\|G\left(\lambda_{0}\right)\right\|\|\Delta\|\|C x\| .
$$

It follows that

$$
\|\Delta\| \geq\left\|G\left(\lambda_{0}\right)\right\|^{-1} \geq\left(\sup _{\lambda \in \overline{\mathbb{C}}^{+}}\|G(\lambda)\|\right)^{-1} .
$$

Case 2: There exists a sequence $\left\{\lambda_{j}\right\}_{j=1}^{\infty}$ such that $\lambda_{j} \in \sigma\left(H_{\Delta}\right)$ and $\operatorname{Re} \lambda_{j}<0$ for all $j$ but $\lim _{n \rightarrow \infty} \operatorname{Re} \lambda_{j}=0$. Then, for all sufficiently large $j$, we have that $\operatorname{Re} \lambda_{j}>\alpha(H)$, which implies $\lambda_{j} \notin \sigma(H)$. Similar to the proof of Case 1, it follows that

$$
\|\Delta\| \geq\left\|G\left(\lambda_{j}\right)\right\|^{-1}
$$

and thus,

$$
\|\Delta\| \geq\left(\sup _{\operatorname{Re} \lambda \geq \operatorname{Re} \lambda_{j}}\|G(\lambda)\|\right)^{-1} .
$$

Since $\|G(\lambda)\|$ is continuous and $\sup _{\operatorname{Re} \lambda \geq 0}\|G(\lambda)\|<\infty$, letting $j \rightarrow \infty$, we obtain

$$
\|\Delta\| \geq\left(\lim _{j \rightarrow \infty} \sup _{\operatorname{Re} \lambda \geq \operatorname{Re} \lambda_{j}}\|G(\lambda)\|\right)^{-1}=\left(\sup _{\operatorname{Re} \lambda \geq 0}\|G(\lambda)\|\right)^{-1},
$$

and the proof is complete.

It is already known for the case of perturbed non-delay DAEs [6], see also [11, that it is necessary to restrict the perturbations in order to get a meaningful concept of the structured stability radius, since a DAE system may lose its regularity and/or stability under infinitesimal perturbations. We therefore introduce the following set of admissible perturbations.

Definition 4.3. Consider a strangeness-free system (1) and let $W \in \mathbb{C}^{n, n}$ be such that (8) holds. A structured perturbation as in (25) is called admissible if (25) is still strangeness-free with the same triple $(d, a, h)$, i.e., there exists a nonsingular $\tilde{W} \in \mathbb{C}^{n, n}$ such that

$$
\begin{aligned}
\tilde{W}^{-1}\left(E+B_{1} \Delta_{1} C\right) & =\left[\begin{array}{c}
\tilde{E}_{1} \\
0 \\
0
\end{array}\right], \tilde{W}^{-1}\left(A+B_{2} \Delta_{2} C\right)=\left[\begin{array}{c}
\tilde{A}_{1} \\
\tilde{A}_{2} \\
0
\end{array}\right], \\
\tilde{W}^{-1}\left(D+B_{3} \Delta_{3} C\right) & =\left[\begin{array}{c}
\tilde{D}_{1} \\
\tilde{D}_{2} \\
\tilde{D}_{3}
\end{array}\right],
\end{aligned}
$$


where $\tilde{E}_{1}, \tilde{A}_{1}, \tilde{D}_{1} \in \mathbb{C}^{d, n}, \tilde{A}_{2}, \tilde{D}_{2} \in \mathbb{C}^{a, n}, \tilde{D}_{3} \in \mathbb{C}^{h, n}$, such that

$$
\left[\begin{array}{c}
\tilde{E}_{1} \\
\tilde{A}_{2} \\
\tilde{D}_{3}
\end{array}\right]
$$

is invertible.

Assume that the matrices $B_{i}, i=1,2,3$, that are restricting the structure have the form

$$
W^{-1} B_{1}=\left[\begin{array}{l}
B_{11} \\
B_{12} \\
B_{13}
\end{array}\right], W^{-1} B_{2}=\left[\begin{array}{l}
B_{21} \\
B_{22} \\
B_{23}
\end{array}\right], W^{-1} B_{3}=\left[\begin{array}{l}
B_{31} \\
B_{32} \\
B_{33}
\end{array}\right],
$$

where $B_{j 1} \in \mathbb{C}^{d, p_{j}}, B_{2 j} \in \mathbb{C}^{a, p_{j}}$, and $B_{3, j} \in \mathbb{C}^{h, p_{j}}, j=1,2,3$. According to $[6$, Lemma 3.3], if the structured perturbation is admissible then $B_{12} \Delta_{1} C=0$, $B_{13} \Delta_{1} C=0$, and $B_{23} \Delta_{2} C=0$. Thus, for the sake of simplicity, we assume that

$$
B_{12}=0, B_{13}=0 \text {, and } B_{23}=0 .
$$

It is easy to see that with all structured perturbations with $B_{i}, i=1,2,3$, satisfying (37), if the perturbation $\Delta$ is sufficiently small, then the strangenessfree property is preserved with the same sizes of the blocks.

We denote the infimum of the norm of all perturbations $\Delta$ such that 25. is no longer strangeness-free or the sizes of the blocks $d, a, h$ change, by $d_{\mathbb{C}}^{s}(E, A, D ; B, C)$, and immediately have the following proposition.

Proposition 4.4. Suppose that equation (1) is strangeness-free and subjected to structured perturbations with $B_{i}, i=1,2,3$ satisfying (37). Then

$$
d_{\mathbb{C}}^{s}(E, A, D ; B, C)=\left\|C\left[\begin{array}{c}
E_{1} \\
A_{2} \\
D_{3}
\end{array}\right]^{-1}\left[\begin{array}{ccc}
B_{11} & 0 & 0 \\
0 & B_{22} & 0 \\
0 & 0 & B_{33}
\end{array}\right]\right\|^{-1}
$$

Proof. With restriction matrices $B_{i}, i=1,2,3$ satisfying (37), the perturbed system $(25)$ is still strangeness-free with $\tilde{E}_{1}, \tilde{A}_{1}, \tilde{D}_{1} \in \mathbb{C}^{d, n}, \tilde{A}_{2}, \tilde{D}_{2} \in$ $\mathbb{C}^{a, n}, \tilde{D}_{3} \in \mathbb{C}^{h, n}$ (as in (35) if and only if

$$
\left[\begin{array}{l}
E_{1}+B_{11} \Delta_{1} C \\
A_{2}+B_{22} \Delta_{2} C \\
D_{3}+B_{33} \Delta_{3} C
\end{array}\right]=\left[\begin{array}{c}
E_{1} \\
A_{2} \\
D_{3}
\end{array}\right]+\left[\begin{array}{ccc}
B_{11} & 0 & 0 \\
0 & B_{22} & 0 \\
0 & 0 & B_{33}
\end{array}\right] \Delta C
$$


is nonsingular. Thus the distance problem is that of the distance of a nonsingular matrix to the nearest singular matrix. For this problem it has been shown, see, e.g., [36], that the matrix

$$
\left[\begin{array}{c}
\tilde{E}_{1} \\
\tilde{A}_{2} \\
\tilde{D}_{3}
\end{array}\right]=\left[\begin{array}{l}
E_{1}+B_{11} \Delta_{1} C_{1} \\
A_{2}+B_{22} \Delta_{2} C_{2} \\
D_{3}+B_{33} \Delta_{3} C_{3}
\end{array}\right]
$$

is nonsingular if

$$
\|\Delta\|<\left\|C\left[\begin{array}{c}
E_{1} \\
A_{2} \\
D_{3}
\end{array}\right]^{-1}\left[\begin{array}{ccc}
B_{11} & 0 & 0 \\
0 & B_{22} & 0 \\
0 & 0 & B_{33}
\end{array}\right]\right\|^{-1}
$$

and the distance to singularity is given by

$$
d_{\mathbb{C}}^{s}(E, A, D ; B, C)=\left\|C\left[\begin{array}{l}
E_{1} \\
A_{2} \\
D_{3}
\end{array}\right]^{-1}\left[\begin{array}{ccc}
B_{11} & 0 & 0 \\
0 & B_{22} & 0 \\
0 & 0 & B_{33}
\end{array}\right]\right\|^{-1} .
$$

Proposition 4.5. Consider system (1) with $\alpha(H)<0$. If the system is strangeness-free and subjected to structured perturbations as in 25 with structure matrices $B_{1}, B_{2}, B_{3}$ satisfying (37) and if the perturbation $\Delta$ satisfies

$$
\|\Delta\|<\left(\sup _{\operatorname{Re} \lambda \geq 0}\|G(\lambda)\|\right)^{-1},
$$

then the structured perturbation is admissable, i.e., the perturbed equation (25) is strangeness-free with the same block-sizes $d, a$, and $h$.

Proof. To prove the assertion, we will show that

$$
\left(\sup _{\operatorname{Re} \lambda \geq 0}\|G(\lambda)\|\right)^{-1} \leq\left\|C\left[\begin{array}{c}
E_{1} \\
A_{2} \\
D_{3}
\end{array}\right]^{-1}\left[\begin{array}{ccc}
B_{11} & 0 & 0 \\
0 & B_{22} & 0 \\
0 & 0 & B_{33}
\end{array}\right]\right\|^{-1}
$$

We can rewrite $G$ as

$$
\begin{aligned}
G(\lambda) & =C H(\lambda)^{-1}\left[\begin{array}{lll}
-\lambda B_{1} & B_{2} & e^{-\lambda \tau} B_{3}
\end{array}\right] \\
& \left.=C\left[\begin{array}{c}
\lambda E_{1}-A_{1}-e^{-\lambda \tau} D_{1} \\
-A_{2}-e^{-\lambda \tau} D_{2} \\
-e^{-\lambda \tau} D_{3}
\end{array}\right]\right]^{-1}\left[\begin{array}{ccc}
-\lambda B_{11} & B_{21} & e^{-\lambda \tau} B_{31} \\
0 & B_{22} & e^{-\lambda \tau} B_{32} \\
0 & 0 & e^{-\lambda \tau} B_{33}
\end{array}\right] \\
& =: C F(\lambda),
\end{aligned}
$$


and thus it follows that

$$
\left[\begin{array}{c}
\lambda E_{1}-A_{1}-e^{-\lambda \tau} D_{1} \\
-A_{2}-e^{-\lambda \tau} D_{2} \\
-e^{-\lambda \tau} D_{3}
\end{array}\right] F(\lambda)=\left[\begin{array}{ccc}
-\lambda B_{11} & B_{21} & e^{-\lambda \tau} B_{31} \\
0 & B_{22} & e^{-\lambda \tau} B_{32} \\
0 & 0 & e^{-\lambda \tau} B_{33}
\end{array}\right]
$$

If $\lambda \neq 0$, then this is equivalent to

$$
\left[\begin{array}{c}
-E_{1}+A_{1} / \lambda+e^{-\lambda \tau} D_{1} / \lambda \\
-A_{2}-e^{-\lambda \tau} D_{2} \\
-D_{3}
\end{array}\right] F(\lambda)=\left[\begin{array}{ccc}
B_{11} & -B_{21} / \lambda & -e^{-\lambda \tau} B_{31} / \lambda \\
0 & B_{22} & e^{-\lambda \tau} B_{32} \\
0 & 0 & B_{33}
\end{array}\right]
$$

and, since

$$
\lim _{\operatorname{Re} \lambda \rightarrow+\infty}\left[\begin{array}{c}
-E_{1}+A_{1} / \lambda+e^{-\lambda \tau} D_{1} / \lambda \\
-A_{2}-e^{-\lambda \tau} D_{2} \\
-D_{3}
\end{array}\right]=-\left[\begin{array}{c}
E_{1} \\
A_{2} \\
D_{3}
\end{array}\right]
$$

and

$$
\lim _{\operatorname{Re} \lambda \rightarrow+\infty}\left[\begin{array}{ccc}
B_{11} & -B_{21} / \lambda & -e^{-\lambda \tau} B_{31} / \lambda \\
0 & B_{22} & e^{-\lambda \tau} B_{32} \\
0 & 0 & B_{33}
\end{array}\right]=\left[\begin{array}{ccc}
B_{11} & 0 & 0 \\
0 & B_{22} & 0 \\
0 & 0 & B_{33}
\end{array}\right],
$$

it follows that $\lim _{\operatorname{Re} \lambda \rightarrow+\infty} F(\lambda)$ exists and

$$
\lim _{\operatorname{Re} \lambda \rightarrow+\infty} F(\lambda)=-\left[\begin{array}{c}
E_{1} \\
A_{2} \\
D_{3}
\end{array}\right]^{-1}\left[\begin{array}{ccc}
B_{11} & 0 & 0 \\
0 & B_{22} & 0 \\
0 & 0 & B_{33}
\end{array}\right]
$$

Thus, it follows that

$$
\lim _{\operatorname{Re} \lambda \rightarrow+\infty} G(\lambda)=C \lim _{\operatorname{Re} \lambda \rightarrow+\infty} F(\lambda)=-C\left[\begin{array}{l}
E_{1} \\
A_{2} \\
D_{3}
\end{array}\right]^{-1}\left[\begin{array}{ccc}
B_{11} & 0 & 0 \\
0 & B_{22} & 0 \\
0 & 0 & B_{33}
\end{array}\right],
$$

and hence (38) holds. It is obvious that

$$
\left(\sup _{\operatorname{Re} \lambda \geq 0}\|G(\lambda)\|\right)^{-1} \leq\left(\lim _{\operatorname{Re} \lambda \rightarrow+\infty}\|G(\lambda)\|\right)^{-1} .
$$

By Proposition 4.4, it follows that if

$$
\|\Delta\|<\left(\sup _{\operatorname{Re} \lambda \geq 0}\|G(\lambda)\|\right)^{-1}
$$

then the perturbed equation 25 is strangeness-free with the same blocksizes $d, a$, and $h$ as for (1). 
We combine these results to characterize the stability radius for strangenessfree DDAEs under suitable structured perturbations.

Theorem 4.6. Suppose that equation (1) is exponentially stable and strangeness-free and subjected to structured perturbations as in (25) with structure matrices $B_{1}, B_{2}, B_{3}$ satisfying (37). Then

$$
r_{\mathbb{C}}(E, A, D ; B, C)=\left(\sup _{\operatorname{Re} \lambda \geq 0}\|G(\lambda)\|\right)^{-1} .
$$

Furthermore, if $\|\Delta\|<r_{\mathbb{C}}(E, A, D ; B, C)$ then (25) is strangeness-free with the same blocksizes $d, a$, and $h$ as for (1).

Proof. By Proposition 4.1, we have

$$
r_{\mathbb{C}}(E, A, D ; B, C) \leq\left(\sup _{\operatorname{Re} \lambda \geq 0}\|G(\lambda)\|\right)^{-1} .
$$

To prove the reverse inequality, let $\Delta$ be an arbitrary perturbation that destroys the exponential stability of equation (1). Assume that

$$
\|\Delta\|<\left(\sup _{\operatorname{Re} \lambda \geq 0}\|G(\lambda)\|\right)^{-1} .
$$

Since equation (1) is strangeness-free and exponentially stable, we have $\alpha(H)<0$ and by Proposition 4.2 , we have also that $\alpha\left(H_{\Delta}\right)<0$. Then by Proposition 4.5 the perturbed equation 25 is strangeness-free, and hence, by Theorem 3.1 we obtain that the perturbed equation 25 is exponentially stable, which is a contradiction. Thus,

$$
\|\Delta\| \geq\left(\sup _{\operatorname{Re} \lambda \geq 0}\|G(\lambda)\|\right)^{-1}
$$

and hence,

$$
r_{\mathbb{C}}(E, A, D ; B, C) \geq\left(\sup _{\operatorname{Re} \lambda \geq 0}\|G(\lambda)\|\right)^{-1},
$$

which implies (39). Finally, by Proposition 4.5 we have that $(25)$ is strangenessfree if $\|\Delta\|<r_{\mathbb{C}}(E, A, D ; B, C)$. 
Remark 4.7. By the maximum principle [28], the supremum of $G(\lambda)$ over the right-half plane is attained at a finite point on the imaginary axis or at infinity. For strangeness-free DDAEs, it can be shown that it suffices to take the supremum of $\|G(\lambda)\|$ over the imaginary axis instead of the whole right-half plane, i.e., we have

$$
r_{\mathbb{C}}(E, A, D ; B, C)=\left(\sup _{\operatorname{Re} \lambda=0}\|G(\lambda)\|\right)^{-1},
$$

see Lemma 5.1 in the Appendix.

As a corollary we obtain the corresponding result for a special case of strangeness-free systems where already the pair $(E, A)$ is regular with $\operatorname{ind}(E, A) \leq 1$.

Corollary 4.8. Consider system (1) with a regular pair (E, A) satisfying $\operatorname{ind}(E, A) \leq 1$ and suppose that the system is exponentially stable and has Weierstraß-Kronecker canonical form (7). If the system is subjected to structured perturbations as in (25), where the structure matrix $B_{1}$ satisfies

$$
W^{-1} B_{1}=\left[\begin{array}{c}
B_{11} \\
0
\end{array}\right]
$$

with $B_{11} \in \mathbb{C}^{d \times p_{1}}$, then the structured stability radius is given by

$$
r_{\mathbb{C}}(E, A, D ; B, C)=\left(\sup _{\operatorname{Re} \lambda=0}\|G(\lambda)\|\right)^{-1} .
$$

For non-delayed DAEs it has been shown [11] that if the perturbation is such that the nilpotent structure in the Weierstraß-Kronecker canonical form is preserved, then one can also characterize the structured stability radius in the case that the pair $(E, A)$ is regular and $\operatorname{ind}(E, A)>1$.

We have seen in Section 3 that exponential stability is characterized by the spectrum of $H$ if we assume that $N D_{21}=0$ and $N D_{22}=0$. In the following we assume that this property is preserved and that in the perturbed equation (25), the structure matrices $B_{1}, B_{2}, B_{3}$ satisfy

$$
W^{-1} B_{1}=\left[\begin{array}{c}
B_{11} \\
0
\end{array}\right], W^{-1} B_{2}=\left[\begin{array}{c}
B_{21} \\
0
\end{array}\right], W^{-1} B_{3}=\left[\begin{array}{c}
B_{31} \\
B_{32}
\end{array}\right], N B_{32}=0
$$

where $B_{j, 1} \in \mathbb{C}^{d, p_{j}}, j=1,2,3, B_{32} \in \mathbb{C}^{n-d, p_{3}}$, and $W \in \mathbb{C}^{n, n}, N \in \mathbb{C}^{n-d, n-d}$ are as in (7). In the following we consider structured perturbations that do 
not alter the nilpotent structure of the Kronecker form (7) of $(E, A)$, i.e., the nilpotent matrix $N$ and the corresponding left invariant subspace associated with eigenvalue $\infty$ is preserved, see [6] for the case that $\operatorname{ind}(E, A)=1$ and $D=0$.

Similar to the approach in [6], we now introduce the distance to the nearest pair with a different nilpotent structure

$d_{\mathbb{C}}^{n}(E, A, D ; B, C)=\inf \{\|\Delta\|: 25)$ does not preserve the nilpotent structure $\}$

Under assumption 40, we obtain the following result, see [11 for the case of non-delay DAEs.

Proposition 4.9. Consider equation (1) with regular $(E, A)$ and $\operatorname{ind}(E, A)>$ 1 , subjected to transformed perturbations satisfying (40). Then the distance to the nearest system with a different nilpotent structure is given by

$$
d_{\mathbb{C}}^{n}(E, A, D ; B, C)=\left\|C_{11} B_{11}\right\|^{-1}
$$

where $C=\left[\begin{array}{ll}C_{11} & C_{12}\end{array}\right]$ with $C_{11} \in \mathbb{C}^{q, r}, C_{12} \in \mathbb{C}^{q, n-r}$.

Proof. With regard to (40), the nilpotent structure of the perturbed equation (25) is preserved if and only if the perturbed matrix $I_{r}+B_{11} \Delta_{1} C_{11}$ is nonsingular. Thus using again the distance of a nonsingular matrix to singularity, see again [36], we obtain

$$
d_{\mathbb{C}}^{n}(E, A, D ; B, C)=\left\|C_{11} B_{11}\right\|^{-1} .
$$

Theorem 4.10. Consider an exponentially stable equation (1) with regular pair $(E, A)$ and $\operatorname{ind}(E, A)>1$ and assume that equation (1) is subjected to transformed perturbations satisfying (40). Then the stability radius is given by the formula

$$
r_{\mathbb{C}}(E, A, D ; B, C)=\left(\sup _{\operatorname{Re} \lambda=0}\|G(\lambda)\|\right)^{-1}
$$

Moreover, if $\|\Delta\|<r_{\mathbb{C}}(E, A, D ; B, C)$ has a regular pair $\left(E+C \Delta_{1} B_{1}, A+\right.$ $\left.C \Delta_{2} B_{2}\right)$ with the same nilpotent structure in the Kronecker canonical form and the perturbed system is exponentially stable. 
Proof. Under the assumption 40, elementary calculations yield

$\lim _{\operatorname{Re} \lambda \rightarrow+\infty}\left\|G_{1}(\lambda)\right\|=\left\|C_{11} B_{11}\right\|, \quad \lim _{\operatorname{Re} \lambda \rightarrow+\infty}\left\|G_{2}(\lambda)\right\|=\lim _{\operatorname{Re} \lambda \rightarrow+\infty}\left\|G_{3}(\lambda)\right\|=0$.

Therefore,

$$
\lim _{\operatorname{Re} \lambda \rightarrow+\infty}\|G(\lambda)\|=\left\|C_{11} B_{11}\right\|
$$

Using the fact that

$$
\sup _{\operatorname{Re} \lambda \geq 0}\|G(\lambda)\| \geq \lim _{\operatorname{Re} \lambda \rightarrow+\infty}\|G(\lambda)\|
$$

and Proposition 4.9, the remainder of the proof is analogous to that of Theorem 4.6. Again by using the maximum principle, it suffices to take the supremum of $\|G(\lambda)\|$ on the imaginary axis instead of the whole right-half plane.

To illustrate the results of this section consider the following example.

Example 4.11. Consider the strangeness-free linear DDAE

$$
\left[\begin{array}{ccc}
1 & 0 & 0 \\
0 & 0 & 0 \\
0 & 0 & 0
\end{array}\right] \dot{x}(t)=\left[\begin{array}{ccc}
-1 & 4 & 0 \\
0 & 2 & 0 \\
0 & 0 & 0
\end{array}\right] x(t)+\left[\begin{array}{ccc}
0 & 2 & 0 \\
0 & 1 & 1 \\
0 & 0 & 1
\end{array}\right] x(t-1)
$$

with singular pair $(E, A)$ subjected to the structured perturbations

$$
\begin{aligned}
& E=\left[\begin{array}{lll}
1 & 0 & 0 \\
0 & 0 & 0 \\
0 & 0 & 0
\end{array}\right] \rightsquigarrow \widetilde{E}=\left[\begin{array}{ccc}
1+\delta_{11} & \delta_{12} & \delta_{13} \\
0 & 0 & 0 \\
0 & 0 & 0
\end{array}\right], \\
& A=\left[\begin{array}{ccc}
-1 & 4 & 0 \\
0 & 2 & 0 \\
0 & 0 & 0
\end{array}\right] \rightsquigarrow \widetilde{A}=\left[\begin{array}{ccc}
-1+3 \delta_{21} & 3 \delta_{22} & 3 \delta_{23} \\
\delta_{21} & 2+\delta_{22} & \delta_{23} \\
0 & 0 & 0
\end{array}\right], \\
& D=\left[\begin{array}{ccc}
0 & 2 & 0 \\
0 & 1 & 1 \\
0 & -1 & 1
\end{array}\right] \rightsquigarrow \widetilde{D}=\left[\begin{array}{ccc}
2 \delta_{31} & 2+2 \delta_{32} & 2 \delta_{33} \\
2 \delta_{31} & 1+2 \delta_{32} & 1+2 \delta_{33} \\
\delta_{31} & \delta_{32} & 1+\delta_{33}
\end{array}\right],
\end{aligned}
$$

which can be represented in the form 25 with

$$
B_{1}=\left[\begin{array}{l}
1 \\
0 \\
0
\end{array}\right], B_{2}=\left[\begin{array}{l}
3 \\
1 \\
0
\end{array}\right], B_{3}=\left[\begin{array}{l}
2 \\
2 \\
1
\end{array}\right], C=I_{3}, \Delta=\left[\begin{array}{lll}
\delta_{11} & \delta_{12} & \delta_{13} \\
\delta_{21} & \delta_{22} & \delta_{23} \\
\hline \delta_{31} & \delta_{32} & \delta_{33}
\end{array}\right]
$$


We have $H(\lambda)=\lambda E-A-e^{-\lambda} D=\left[\begin{array}{ccc}1+\lambda & -2\left(2+e^{-\lambda}\right) & 0 \\ 0 & -2-e^{-\lambda} & -e^{-\lambda} \\ 0 & 0 & -e^{-\lambda}\end{array}\right]$, and it is easy to check that $\alpha(H)<0$ and therefore (41) is exponentially stable.

By simple computations, we get

$$
G(\lambda)=\left[\begin{array}{ccc}
\frac{-\lambda}{1+\lambda} & \frac{1}{1+\lambda} & 0 \\
0 & \frac{-1}{2+e^{-\lambda}} & \frac{-e^{-\lambda}}{2+e^{-\lambda}} \\
0 & 0 & -1
\end{array}\right]
$$

and with $\|\cdot\|$ being the maximum norm of $\mathbb{C}^{3}$, it follows that

$$
\sup _{\lambda \in i \mathbb{R}}\|G(\lambda)\|_{\infty}=\|G(i \pi)\|_{\infty}=2 .
$$

Thus, by Theorem 4.6, we obtain

$$
r_{\mathbb{C}}(E, A, D ; B, C)=\frac{1}{\sup _{\lambda \in i \mathbb{R}}\|G(\lambda)\|_{\infty}}=\frac{1}{2} .
$$

We note that by using (30), a destabilizing perturbation is easily constructed as

$$
\Delta=\left[\begin{array}{ccc}
0 & 0 & 0 \\
0 & -1 / 2 & 0 \\
0 & 1 / 2 & 0
\end{array}\right]
$$

with norm $1 / 2$. Further, one can easily check that with this $\Delta$ the perturbed system remains strangeness-free, but $\alpha\left(H_{\Delta}\right)=0$, which means that the perturbed system is not asymptotically stable.

\section{Conclusion}

Characterizations for exponential stability and robust exponential stability of DDAEs have been derived under the assumption that the coefficient matrices are subjected to structured perturbations. The spectral condition for exponential stability has been investigated in the class of strangeness-free DDAEs as well as higher index DDAEs. Formulas for the complex stability radius and the class of allowable perturbations for DDAEs have been derived in both cases. However, the validity of a spectral condition for the exponential stability of DDAEs in the general case and formulas for the real stability radius of DDAEs are still open problems. 


\section{References}

[1] U. M. Ascher and L. R. Petzold, The numerical solution of delaydifferential algebraic equations of retarded and neutral type, SIAM J. Numer. Anal. 32 (1995), 1635-1657.

[2] C.T.H. Baker, C.A.H. Paul, and H. Tian, Differential algebraic equations with after-effect, J. Comp. Appl. Math. 140 (2002), 63-80.

[3] A. Bellen and M. Zennaro, Numerical methods for delay differential equations, Oxford University Press, Oxford, UK, 2003.

[4] K. E. Brenan, S. L. Campbell, and L. R. Petzold, Numerical solution of initial-value problems in differential algebraic equations, 2nd ed., SIAM Publications, Philadelphia, PA, 1996.

[5] R. Byers, C. He, and V. Mehrmann, Where is the nearest non-regular pencil, Lin. Alg. Appl. 285 (1998), 81-105.

[6] R. Byers and N.K. Nichols, On the stability radius of a generalized state-space system, Lin. Alg. Appl. 188-189 (1993), 113-134.

[7] S. L. Campbell, Singular systems of differential equations I, Pitman, San Francisco, CA, 1980.

[8] S. L. Campbell, 2-D (differential-delay) implicit systems, Proc. IMACS World Congress on Scientific Computation, Dublin (1991), 1828-1829.

[9] S. L. Campbell, Nonregular 2D descriptor delay systems, IMA J. Math. Control Appl. 12 (1995), 57-67.

[10] S.L. Campbell and V.H. Linh, Stability criteria for differential-algebraic equations with multiple delays and their numerical solutions, Appl. Math. Comp. 208 (2009), 397-415.

[11] N.H. Du, V. H. Linh, and V. Mehrmann, Robust stability of differentialalgebraic equations, in: Surveys in Differential-Algebraic Equations I, DAE-F (2013), 63-95.

[12] N.H. Du, D.D. Thuan, and N.C. Liem, Stability radius of implicit dynamic equations with constant coefficients on time scales, Systems Control Lett. 60 (2011), 596-603.

[13] E. Fridman, Stability of linear descriptor systems with delay: a Lyapunov-based approach, J. Math. Anal. Appl. 273 (2002), 24-44. 
[14] F. R. Gantmacher, The theory of matrices II, Chelsea Publishing Company, New York, NY, 1959.

[15] E. Griepentrog and R. März, Differential-algebraic equations and their numerical treatment, Teubner Verlag, Leipzig, Germany, 1986.

[16] N. Guglielmi and E. Hairer, Computing breaking points in implicit delay differential equations, Adv. Comp. Math. 29 (2008), 229-247.

[17] P. Ha and V. Mehrmann, Analysis and reformulation of linear delay differential-algebraic equations, Elect. J. Linear Algebra 23 (2012), 703730 .

[18] P. Ha, V. Mehrmann, and A. Steinbrecher, Analysis of linear variable coefficient delay differential-algebraic equations, in preparation, 2013.

[19] E. Hairer and G. Wanner, Solving ordinary differential equations II: Stiff and differential-algebraic problems, 2nd ed., Springer-Verlag, Berlin, Germany, 1996.

[20] J.K. Hale, Theory of functional-differential equations, Springer-Verlag, New York-Heidelberg-Berlin, 1977.

[21] D. Hinrichsen and A. J. Pritchard, Stability radii of linear systems, Systems Control Lett. 7 (1986), 1-10.

[22] D. Hinrichsen and A. J. Pritchard, Stability radius for structured perturbations and the algebraic riccati equation, Systems Control Lett. 8 (1986), 105-113.

[23] D. Hinrichsen and A. J. Pritchard, Mathematical systems theory I. Modelling, state space analysis, stability and robustness, Springer-Verlag, New York, NY, 2005.

[24] G. Hu and E. J. Davison, Real stability radii of linear time-invariant time-delay systems, Systems Control Lett. 50 (2003), 209-219.

[25] P. Kunkel and V. Mehrmann, Differential-algebraic equations. Analysis and numerical solution, EMS Publishing House, Zürich, Switzerland, 2006.

[26] P. Kunkel and V. Mehrmann, Stability properties of differentialalgebraic equations and spin-stabilized discretization, Electr. Trans. Num. Anal. 26 (2007), 383-420. 
[27] R. Lamour, R. März, and C. Tischendorf, Differential-Algebraic Equations: A Projector Based Analysis, Springer, 2013.

[28] S. Lang, Complex Analysis, Springer, 1999.

[29] V.H. Linh and V. Mehrmann, Lyapunov, Bohl and Sacker-Sell spectral intervals for differential-algebraic equations, J. Dynamics Diff. Eq. 21 (2009), 153-194.

[30] T. Luzyanina and D. Rose, Periodic solutions of differential-algebraic equations with time-delays: computation and stability analysis, J. Bifurcation Chaos 16 (2006), 67-84.

[31] W. Michiels, Spectrum-based stability analysis and stabilisation of systems described by delay differential algebraic equations, IET Control Theory Appl. 5 (2011), 1829-1842.

[32] P.C. Müller, On the stability of linear descriptor systems by applying modified lyapunov equations, Proceed. Appl. Math. Mech., PAMM 3 (2003), 136-137.

[33] R. Riaza, Differential-algebraic systems. Analytical aspects and circuit applications, World Scientific Publishing Co. Pte. Ltd., Hackensack, NJ., 2008.

[34] L.F. Shampine and P. Gahinet, Delay-differential-algebraic equations in control theory, Appl. Numer. Math. 56 (2006), 574-588.

[35] N.K. Son and P.H.A. Ngoc, Stability radius of linear delay systems, Proceedings of the American Control Conference, San Diego, California (June 1999), 815-816.

[36] N.K. Son and D.D. Thuan, On the radius of surjectivity for rectangular matrices and its application to measuring stabilizability of linear systems under structured perturbations, J. Nonlin. Convex Anal. 12 (2011), 441-453.

[37] T. Stykel, Analysis and numerical solution of generalized Lyapunov equations, Dissertation, Institut für Mathematik, TU Berlin, Berlin, Germany, 2002.

[38] T. Stykel, On criteria for asymptotic stability of differential-algebraic equations, Z. Angew. Math. Mech. 92 (2002), 147-158. 
[39] H. Tian, Q. Yu, and J. Kuang, Asymptotic stability of linear neutral delay differential-algebraic equations and linear multistep methods, SIAM J. Numer. Anal 49 (2011), 608-618.

[40] J. Wei, Eigenvalue and stability of singular differential delay systems, J. Math. Anal. Appl. 297 (2004), 305-316.

[41] S. Xu, P. Van Dooren, S. Radu, and J. Lam, Robust stability and stabilization for singular systems with state delay and parameter uncertainty, IEEE Trans. Automat. Control 47 (2002), 1122-1128.

[42] Q.C. Zhong, Robust control of time-delay systems, Springer, 2006.

[43] W. Zhu and L.R. Petzold, Asymptotic stability of linear delay differential-algebraic equations and numerical methods, Appl. Numer. Math. 24 (1997), 247-264.

[44] W. Zhu and L.R. Petzold, Asymptotic stability of Hessenberg delay differential-algebraic equations of retarded or neutral type, Appl. Numer. Math. 27 (1998), 309-325.

\section{Appendix}

In this appendix we give a proof for the statement in Remark 4.7 which is stated as the following lemma.

Lemma 5.1. Under the conditions of Theorem 4.6 or Corollary 4.8, we have

$$
\sup _{\operatorname{Re} \lambda \geq 0}\|G(\lambda)\|=\sup _{\operatorname{Re} \lambda=0}\|G(\lambda)\|,
$$

where $G$ is defined in (28).

Proof. Since $G(\lambda)$ is analytic in the right half of the complex plane, by the maximum principle, the supremum of $\|G(\lambda)\|$ is attained on the boundary, that is either on the imaginary axis or somewhere at infinity. It remains to show that the supremum is indeed attained on the imaginary axis (either at a finite point or at infinity).

i) Let us first consider the case that $\operatorname{ind}(E, A) \leq 1$ and that the system is in Weierstraß-Kronecker canonical form (7). We then have

$$
H(\lambda)^{-1}=T\left[\begin{array}{cc}
\lambda I-J-D_{11} e^{-\lambda \tau} & -D_{12} e^{-\lambda \tau} \\
-D_{21} e^{-\lambda \tau} & -I-D_{22} e^{-\lambda \tau}
\end{array}\right]^{-1} W^{-1}
$$


Since for sufficiently large $|\lambda|, \lambda I-J-D_{11} e^{-\lambda \tau}$ is invertible, we can apply the inversion formula for block matrices $M$ of the form

$$
M=\left[\begin{array}{ll}
M_{11} & M_{12} \\
M_{21} & M_{22}
\end{array}\right]
$$

with $M_{11}=\lambda I-J-D_{11} e^{-\lambda \tau}, M_{12}=-D_{12} e^{-\lambda \tau}, M_{21}=-D_{21} e^{-\lambda \tau}$, and $M_{22}=-I-D_{22} e^{-\lambda \tau}$, and the inverse is given by

$M^{-1}=\left[\begin{array}{cc}I & -M_{11}^{-1} M_{12} \\ 0 & I\end{array}\right]\left[\begin{array}{cc}M_{11}^{-1} & 0 \\ 0 & \left(M_{22}-M_{21} M_{11}^{-1} M_{12}\right)^{-1}\end{array}\right]\left[\begin{array}{cc}I & 0 \\ -M_{21} M_{11}^{-1} & I\end{array}\right]$.

Moreover, $\lim _{|\lambda| \rightarrow \infty}\left(\lambda I-J-D_{11} e^{-\lambda \tau}\right)^{-1}=0$ and $\lim _{|\lambda| \rightarrow \infty} \lambda(\lambda I-J-$ $\left.D_{11} e^{-\lambda \tau}\right)^{-1}=I$. Therefore, for all $\varepsilon>0$, there exists $L>0$ such that for $\lambda$ satisfying $|\lambda| \geq L$ and $\operatorname{Re} \lambda \geq 0$, we have

$$
\|G(\lambda)-\tilde{G}(\lambda)\| \leq \varepsilon
$$

where

$$
\tilde{G}(\lambda)=\left[\begin{array}{lll}
\tilde{G}_{1}(\lambda) & \tilde{G}_{2}(\lambda & \tilde{G}_{3}(\lambda)
\end{array}\right]
$$

with

$$
\tilde{G}_{1}(\lambda)=C T\left[\begin{array}{ll}
I & 0 \\
0 & 0
\end{array}\right]\left[\begin{array}{c}
B_{11} \\
0
\end{array}\right], \tilde{G}_{2}(\lambda)=C T\left[\begin{array}{cc}
0 & 0 \\
0 & -\left(I+D_{22} e^{-\lambda \tau}\right)^{-1}
\end{array}\right]\left[\begin{array}{l}
B_{21} \\
B_{22}
\end{array}\right],
$$

and

$$
\tilde{G}_{3}(\lambda)=e^{-\lambda \tau} C T\left[\begin{array}{cc}
0 & 0 \\
0 & -\left(I+D_{22} e^{-\lambda \tau}\right)^{-1}
\end{array}\right]\left[\begin{array}{l}
B_{31} \\
B_{32}
\end{array}\right] .
$$

By introducing a new variable $z=e^{-\lambda \tau}$, since $\operatorname{Re} \lambda \geq 0$, we have $|z| \leq 1$. By the maximum principle, the supremum of $\tilde{G}$ as a function of $z$ over the disk $|z| \leq 1$ is attained on the boundary $|z|=1$, or equivalently

$$
\sup _{\operatorname{Re} \lambda \geq 0,|\lambda| \geq L}\|\tilde{G}(\lambda)\|=\sup _{\operatorname{Re} \lambda=0,|\lambda| \geq L}\|\tilde{G}(\lambda)\| .
$$

Because of (44), we have

$$
\begin{aligned}
\sup _{\operatorname{Re} \lambda \geq 0,|\lambda| \geq L}\|G(\lambda)\| & \leq \sup _{\operatorname{Re} \lambda \geq 0,|\lambda| \geq L}\|\tilde{G}(\lambda)\|+\varepsilon \\
& =\sup _{\operatorname{Re} \lambda=0,|\lambda| \geq L}\|\tilde{G}(\lambda)\|+\varepsilon \\
& \leq \sup _{\operatorname{Re} \lambda=0,|\lambda| \geq L}\|G(\lambda)\|+2 \varepsilon .
\end{aligned}
$$


Analogously, we have

$$
\sup _{\operatorname{Re} \lambda \geq 0,|\lambda| \geq L}\|G(\lambda)\| \geq \sup _{\operatorname{Re} \lambda=0,|\lambda| \geq L}\|G(\lambda)\|-2 \varepsilon .
$$

On the other hand

$$
\sup _{\operatorname{Re} \lambda \geq 0,|\lambda| \leq L}\|G(\lambda)\|=\max \left\{\sup _{\operatorname{Re} \lambda=0,|\lambda| \leq L}\|G(\lambda)\|, \sup _{\operatorname{Re} \lambda \geq 0,|\lambda|=L}\|G(\lambda)\|\right\} .
$$

Hence,

$$
\sup _{\operatorname{Re} \lambda=0}\|G(\lambda)\|-2 \varepsilon \leq \sup _{\operatorname{Re} \lambda \geq 0}\|G(\lambda)\| \leq \sup _{\operatorname{Re} \lambda=0}\|G(\lambda)\|+2 \varepsilon .
$$

Since $\varepsilon$ is arbitrary, the identity 42 holds.

ii) For the general case of a strangeness-free system of the form (1) that it is transformed into the form (8) and that satisfies (37) we have

$$
\begin{aligned}
H(\lambda)^{-1}= & {\left[\begin{array}{c}
\lambda E_{1}-A_{1}-e^{-\lambda \tau} D_{1} \\
-A_{2}-e^{-\lambda \tau} D_{2} \\
-e^{-\lambda \tau} D_{3}
\end{array}\right]^{-1} W^{-1} } \\
= & {\left[\begin{array}{c}
\lambda E_{1}-A_{1}-e^{-\lambda \tau} D_{1} \\
-A_{2}-e^{-\lambda \tau} D_{2} \\
-D_{3}
\end{array}\right]^{-1}\left[\begin{array}{ccc}
I & 0 & 0 \\
0 & I & 0 \\
0 & 0 & e^{\lambda \tau}
\end{array}\right] W^{-1} . }
\end{aligned}
$$

Taking into account (37), we obtain

$$
\begin{aligned}
& G_{1}(\lambda)=\lambda\left[\begin{array}{c}
\lambda E_{1}-A_{1}-e^{-\lambda \tau} D_{1} \\
-A_{2}-e^{-\lambda \tau} D_{2} \\
-D_{3}
\end{array}\right]^{-1}\left[\begin{array}{c}
B_{11} \\
0 \\
0
\end{array}\right], \\
& G_{2}(\lambda)=\left[\begin{array}{c}
\lambda E_{1}-A_{1}-e^{-\lambda \tau} D_{1} \\
-A_{2}-e^{-\lambda \tau} D_{2} \\
-D_{3}
\end{array}\right]^{-1}\left[\begin{array}{c}
B_{21} \\
B_{22} \\
0
\end{array}\right],
\end{aligned}
$$

and

$$
G_{3}(\lambda)=\left[\begin{array}{c}
\lambda E_{1}-A_{1}-e^{-\lambda \tau} D_{1} \\
-A_{2}-e^{-\lambda \tau} D_{2} \\
-D_{3}
\end{array}\right]^{-1}\left[\begin{array}{c}
e^{-\lambda \tau} B_{31} \\
e^{-\lambda \tau} B_{32} \\
B_{33}
\end{array}\right]
$$

The assumption that the system is strangeness-free implies that the matrix pair

$$
\left[\begin{array}{c}
E_{1} \\
0 \\
0
\end{array}\right],\left[\begin{array}{c}
A_{1} \\
A_{2} \\
D_{3}
\end{array}\right]
$$


is of index-1 and thus the claim follows by analogous arguments as in Part i). 